\title{
Electrochemical sensors between the academic world and harsh reality: a few thoughts on the past, present, and future
}

\author{
Vladimir M. Mirsky ${ }^{1}$ (D) \\ Received: 2 June 2020 / Revised: 2 June 2020 / Accepted: 4 June 2020 / Published online: 14 June 2020 \\ (C) The Author(s) 2020
}

\author{
"There are three sides to every story. Yours, mine, and \\ the cold hard truth." \\ Unknown author
}

The development of electrochemical sensors and electroanalytical methods had a strong impact on the development of electrochemistry but also on other fields of science. The discovery of polarography has influenced the development of electrochemistry for a few decades. The invention of $\mathrm{pH}$ electrode was the main motivation in the formulation and development of the whole concept of chemical sensors. However, either due to geniality of Fritz Haber or because of the sarcasm of nature, even after over 100 years of the development, no single sensor with so unique analytical properties (selectivity, dynamic range, long-time stability) as $\mathrm{pH}$ electrode was developed. Although many types of chemical sensors became necessary components of our usual life or industrial processes, the real progress measured in the number of new devices and concepts realized as a commercial product is relatively slow.

Not all unsolved problems of electrochemical sensors have an electrochemical nature. For example, the development of ion-selective electrodes is strongly dependent on the progress in molecular design and organic synthesis of corresponding ionophores. Very similar limitations define the development of selective electrocatalytical sensors and especially electrochemical affinity sensors, and also in these cases, there is a lack of chemical receptors with high affinity and selectivity. But in general, the number of known chemical receptors is very limited, and only very few of them possess high values

Vladimir M. Mirsky

mirsky@b-tu.de

1 Nanobiotechnology Department, Institute of Biotechnology, Brandenburg University of Technology Cottbus - Senftenberg, 01968 Senftenberg, Germany of the binding constant [1]. A promising approach in this field is the implementation of cyclic molecular evolution consisting of (i) a combinatorial organic synthesis, (ii) a high-throughput screening of the property to optimize, and (iii) data analysis (usually by using of neuronal networks) to design the next evolution cycle [2]. We tried to apply such approach for the development of affinity sensors based on conducting polymers [3], but an effective implementation of this strategy requires a formation of large scientific consortiums with extensive funding.

Obviously, each general combinatorial approach for development of new materials with high affinity using standard protocols and allowing one to be realized without large investments always is the goal of intensive investigation. Two such concepts were suggested. The first one is the concept of molecularly imprinted polymerization [4], first mentioned in the 1930 s, rediscovered in the 1980 s, and being intensively studied since the end of the 1990s. The technology is based on the following steps: (i) formation of complexes between a template (analyte) molecule and complementary monomers, (ii) cross-linking polymerization of these monomers in the presence of porogens, and (iii) extraction of the template molecules. However, such problems like a relatively poor affinity (in most cases below $10^{7} \mathrm{~L} / \mathrm{mol}$ [1]) and strong heterogeneity of the binding sites compromising the binding selectivity, complicate applications of molecularly imprinted polymers in sensor devices. The second concept is based on the exploitation of affinity of nucleic acids (DNA or RNA), sometimes with terminal modification by other chemical groups. The development of such molecules (aptamers) is performed by the procedure of systematic evolution of ligands by exponential enrichment (SELEX) which includes a synthesis of randomized oligonucleotide sequences, selection of the molecules with highest affinity, and their further biosynthesis using polymerase chain reaction. Recently, this approach was extended to peptides, and here, the complexes of peptides with corresponding oligonucleotides were used for PCR 
amplification of the selected peptides [5]. However, also by this technology, it is really challenging to obtain aptamers with high affinity [6]. But the development continues, and one can hope for new ideas and further increase of optimism.

Another problem in the development of selective and sensitive affinity sensors is a very slow sensor recovery. This drawback may be sound to be too academic, but actually, this is the only reason why many highly sensitive (electro)chemical sensors did not find practical applications. Physically, the affinity is defined by the ratio of kinetic constants of analyte binding and dissociation. Therefore, in many cases, a high affinity to the analyte (which is required not only for a high sensitivity but also for a high selectivity of the sensor device) is achieved by minimization of the kinetic constant of the analyte dissociation. The sensor recovery can be accelerated by heating but it is hardly applicable for liquid analytes. A novel approach is based on electrochemical control of the redox state of the sensor layer [7, 8]. Currently, an application of this approach was limited only to chemical sensors based on conducting polymers, but it can be certainly extended to other redox-active receptors. One can expect further progress in this field by introducing of redox-active groups into other receptor molecules (various chemical receptors, nucleic acids, aptamers, or even native antibodies): this may be a way to achieve electrochemically controlled affinity of these receptors.

Due to the strong pressure of the market and costs, the development of sensors was almost focused on a single measurement technology. This led to competitive consideration of different approaches, and during the last decade, we always observed such competitions, for example, Clark electrode vs. fluorescence quenching in oxygen sensing. Simultaneous application of two or more different measurement technologies was used only in science but was not typical for final devices. The cost decrease of the measurement technologies leads to the change of this paradigm, and we observe now such sensors where a high synergetic potential of application of different technologies (usually optical and electrochemical) is exploited. One example of this friendship is the establishing of electroluminescent techniques. Recently, this trend was extended to the new challenge in electrochemical analytics - detection of nanoparticles. Using microelectrodes, it was possible to perform electrochemical analysis of individual nanoparticles [9], and this resulted in the concept of "impact electrochemistry." However, the requirement to measure fast current kinetics leads to the necessity to have a small electrode capacitance (this capacitance is included into the feedback of the current amplifier, thus limiting time resolution). This leads to the fundamental limitation of the electrode area and requires a high particle concentration for the analysis. The use of optical reading of electrochemical conversions of metallic nanoparticles allows one to make an electrochemical analysis of individual nanoparticles on macroscopic electrode surface where hundreds thousands of nanoparticles are present [10]. Notably, this reading of the electrochemical conversion is performed simultaneously for all nanoparticles adsorbed on the electrode surface. Another example of successful implementation of optical techniques in electrochemical sensors is a photomodulation of electrochemical processes using quantum dots [11]. Certainly, the synergetic combination of optical and electrochemical measurement technologies has a high potential for further development of electrochemical (or more exactly - optoelectrochemical) sensors.

Finally, one has to mention a specific problem in the publications on electrochemical sensors. The huge amount of submitted manuscripts in this field describes "new sensors" obtained by an application of a cocktail from some "hot" materials (like nanoparticles, graphene, nanotubes, quantum dots) for electrocatalytical detection of any (typically-relatively exotic) analyte. Only very few of these manuscripts are based on scientific ideas exploiting unique properties of nanomaterials, while the majority presents rather phenomenological observations without any electrochemical study of the effect or/and constructive and systematic comparison of different composites. Unfortunately, many such manuscripts pass through peer-reviewed filter. Let us hope that this nonscientific problem of modern science will be solved already in the near future.

Acknowledgments The author thanks Prof. Fritz Scholz for the invitation to share his thoughts on some aspects of future electrochemical research.

Funding information Open Access funding provided by Projekt DEAL.

Open Access This article is licensed under a Creative Commons Attribution 4.0 International License, which permits use, sharing, adaptation, distribution and reproduction in any medium or format, as long as you give appropriate credit to the original author(s) and the source, provide a link to the Creative Commons licence, and indicate if changes were made. The images or other third party material in this article are included in the article's Creative Commons licence, unless indicated otherwise in a credit line to the material. If material is not included in the article's Creative Commons licence and your intended use is not permitted by statutory regulation or exceeds the permitted use, you will need to obtain permission directly from the copyright holder. To view a copy of this licence, visit http://creativecommons.org/licenses/by/4.0/.

\section{References}

1. Yatsimirsky AK, Mirsky VM (2011) Quantitative affinity data on selected artificial receptors. In: Yatsimirsky AK, Mirsky VM (eds) Artificial receptors for chemical sensors. Wiley-VCH, Weinheim

2. Potyrailo RA, Mirsky VM (eds) (2009) Combinatorial methods for chemical and biological sensors. Springer

3. Mirsky VM, Kulikov V, Hao Q, Wolfbeis OS (2004) Multiparameter high throughput characterization of combinatorial 
chemical microarrays of chemosensitive polymers. Macromol Rap Comm 25(1):253-258

4. Chen L, Wang X, Lu W, Wu X, Li J (2016) Molecular imprinting: perspectives and applications. Chem Soc Rev 45:2137-2211. https://doi.org/10.1039/c6cs00061d

5. Mascini M, Palchetti I, Tombelli S (2012) Nucleic acid and peptide aptamers: fundamentals and bioanalytical aspects. Angew Chemie 51:1316-1332. https://doi.org/10.1002/anie.201006630

6. Haßel SK, Mayer G (2019) Aptamers as therapeutic agents: has the initial euphoria subsided? Molec Diag Therapy 23(3):301-309. https://oi.org/10.1007/s40291-019-00400-6

7. Lange U, Mirsky VM (2011) Integrated electrochemical transistor as a fast recoverable gas sensor. Anal Chim Acta 687(1):7-11. https://doi.org/10.1016/j.aca.2010.12.008

8. Efremenko Y, Mirsky VM (2016) Electrically controlled variation of receptor affinity. Anal Bioanal Chem 408(26):7283-7287. https://doi.org/10.1007/s00216-016-9751-1
9. Sokolov SV, Eloul S, Kätelhön E, Batchelor-McAuleya C, Compton RG (2017) Electrode-particle impacts:a users guide. Phys Chem Chem Phys 19(1):28-43. https://doi.org/10.1039/ C6CP07788A

10. Nizamov S, Kasian O, Mirsky VM (2016) Individual detection and electrochemically assisted identification of adsorbed nanoparticles by using surface plasmon microscopy. Angew. Chemie, 55:7247 $7251 \mathrm{https} / / /$ doi.org/10.1002/anie.201600853

11. Yue Z, Lisdat F, Parak WJ, Hickey SG, Tu L, Sabir N, Dorfs D, Bigall NC (2013) Quantum-dot-based photoelectrochemical sensors for chemical and biological detection. ACS Appl Mater Interfaces 5:2800-2814. https://doi.org/10.1021/am3028662

Publisher's note Springer Nature remains neutral with regard to jurisdictional claims in published maps and institutional affiliations. 